\title{
Poly(amic Acid) and Polyimide Characterization Using Gas Chromatography/Mass Spectrometry and Particle Beam Liquid Chromatography/Mass Spectrometry
}

\author{
Timothy MacMahon \\ Department of Science and Engineering, Orange County Community College, Middletown, New York, USA \\ Mark Chace \\ IBM Corporation, Hopewell Junction, New York, USA
}

\begin{abstract}
A number of polymers were hydrolyzed in $\mathrm{NH}_{4} \mathrm{OH}$ and studied using gas chromatography/ mass spectrometry (GC/MS) and particle beam liquid chromatography/mass spectrometry (particle beam LC/MS) techniques. The polymers studied in this report were as follows: BPDA-PDA, BPDA-PDA-ODA, BPDA-PDA-6FDA, PMDA-ODA, and BTDA-APB. Some of the polymer samples were hydrolyzed in both their acid and imide forms to see if any mass spectrometric differences could be detected. In all cases, the acid and imide spectra looked the same. GC/MS was unable to determine either the amine or acid portion of these polymers via a direct injection of the sample, but when the samples were first extracted with diethyl ether and this ether extract was injected into the chromatograph, the amine portion of the polymers was readily detected. The acid portion was, again, not detected in either the sample or the ether extract. The particle beam was able to detect both the amine and acid monomeric units in the nonextracted sample. (J Am Soc Mass Spectrom 1994, 5, 299-304)
\end{abstract}

$\mathrm{T}$ The analysis of polyimides is becoming increasingly more inportant as this class of polynlers becomes more prevalent in today's electronic and packaging technology [1]. Polyimides are noted for their high glass transition temperatures and thermal stability. In some cases, this thermal stability allows heating to temperatures as high as $400{ }^{\circ} \mathrm{C}$ for many hours without a critical drop in physical or mechanical properties [1]. In addition to the use of polyimides as dielectric layers, there is also interest in developing photosensitive polyimides that could be used as both the lithographic material and insulating dielectric; thus, unlike resists currently in use, the new polyimide resists would not have to be removed after lithography, thereby saving numerous processing steps and shortening cycle times.

The high thermal stability of these polymers makes them difficult to analyze by traditional analytical techniques [1]. There have been a few mass spectrometric studies of this class of polymer. Johnson [2] looked at BTDA-PDA-ODA copolymer using pyrolysis mass spectrometry and saw only water and solvent outgassing up to $350{ }^{\circ} \mathrm{C}$. At $1400^{\circ} \mathrm{C}$, the polymer showed

Address reprint requests to: Dr. Timothy MacMahon, Department of Science and Engineering, Orange County Community College, 115 South Street, Middletown, NY 10940. many breakdown products which were not easily relatable to the bulk polymer. This complexity was attributed to the large population of reactive radical species generated during the pyrolysis experiment. Goldfarb and Jones [3] used a similar technique, TGAMS, where they heated an unknown polyimide to 1000 ${ }^{\circ} \mathrm{C}$ and, as in the previous case, had complex spectra which were difficult to interpret. Laser desorption has also been tried on this class of polymer. Otis [4] looked at PMDA-ODA films using an excimer laser for desorption followed by multiphoton ionization of the desorbed neutrals in a time-of-flight apparatus. The product distribution was, as one might expect, very dependent on the wavelength of the ionizing radiation but in no case were the detected ions simple cleavage products of the parent polymer. The ionic fragments from laser desorption have also been studied by Creasy and Brenna [5] using an Nd:YAG laser, and again the fragmentation did not easily relate to the bulk polymer. Finally, static secondary ionization mass spectrometry (SIMS) using time-of-flight detection [6] has been used to study the surface of a few polyimides. This technique was able to get some structural and surface bonding information and will be discussed later in this article.

Gas chromatography/mass spectrometry (GC/MS) has also been used to characterize these polymers, but 
first the polymer had to be degraded. Chace and Plat [7] developed a mass spectrometric technique based on an older method by Linde and Meeks [8] which uses a hydrolysis reaction to totally hydrolyze the polymer. Once the hydrolysis is complete, information about the amine and acid portion of the molecule can be probed via mass spectrometry. The amine portion can be detected via an ethyl ether extract, but to detect the acids their volatility must first be increased through derivatization to the methyl ester or the trimethylsilyl ester, making them more amenable to GC/MS.

The advent of liquid chromatography/mass spectrometry (LC/MS) opened up new capabilities in the analysis of these polyimides. Particle beam LC/MS can be used on molecules of low volatility and /or thermal instability because separation is achieved in the liquid not in the gaseous phase; thus, operating temperatures are considerably lower [9].

\section{Experimental}

There were two mass spectrometers used in this study. The first was an ITS-40 gas chromatograph/mass spectrometer from Finnigan MAT (San Jose, CA). It is equipped with a $30 \mathrm{~m}$ DB-1 capillary column (i.d. 0.25 $\mathrm{mm}$ ) which is a $25 \mu \mathrm{m}$ polydimethylsiloxane column. GC conditions consisted of a $70{ }^{\circ} \mathrm{C}$ to $300{ }^{\circ} \mathrm{C}$ at 10 ${ }^{\circ} \mathrm{C} / \mathrm{min}$ ramp; chromatographic conditions were the same for all samples and components were identified based on retention time and mass spectra.

The second mass spectrometer is a Kratos MS25RFA sector mass spectrometer (Kratos Analytical, Ramsey, NI) scanned at $1 \mathrm{sec} /$ decade with the source kept at $200{ }^{\circ} \mathrm{C}$ and the acceleration voltage was $4000 \mathrm{~V}$ for all experiments. All spectra were acquired in the standard electron impact mode with $70 \mathrm{eV}$ as the ionization potential. The liquid chromatograph was an IBM Instruments 9650 ternary gradient liquid chromatograph, and methanol was used throughout these experiments as the mobile phase at a flow rate of $1 \mathrm{~mL} / \mathrm{min}$. Although an LC system was used, no attempt at separation was performed. The LC unit was used exclusively to pump the mobile phase, thus spectra were acquired in the flow injection analysis mode of the particle beam interface.

The polymeric monomers used in this study were as follows: $\mathrm{BPDA}=3,3^{\prime}, 4,4^{\prime}$-biphenyltetracarboxylic dianhydride; $\mathrm{PDA}=$ phenylene diamine; $\mathrm{ODA}=4,4^{\prime}$ oxydianiline; $6 \mathrm{FDA}=2,2-\mathrm{b}$ is $(4$-am inophenyl) hexafluoropropane; PMDA $=1,2,4,5$-benzenetetracarboxylic dianhydride (pyromellitic dianhydride); BTDA $=3,3^{\prime}, 4,4^{\prime}$-benzophenonetetracarboxylic dianhydride; $\mathrm{APB}=1,3$-bis (4-aminophenoxy)benzene. (For the individual polymeric structures, see the appropriate figures throughout the text.)

Hydrolysis of the polyimides was accomplished in aqueous ammonium hydroxide $(\sim 40 \%)$. Approximately $5 \mathrm{mg}$ of polymer was added to $2 \mathrm{~mL}$ of ammonium hydroxide and heated at $80^{\circ} \mathrm{C}$ in a closed system to prevent the ammonium from evaporating until digestion was complete (24-48 hours). The samples were allowed to slowly evaporate, at about $80{ }^{\circ} \mathrm{C}$, to about one half their original volume to concentrate the sample and minimize the amount of unreacted $\mathrm{NH}_{4} \mathrm{OH}$. These solutions were filtered and run directly into the particle beam liquid chromatograph/mass spectrometer or extracted with diethyl ether for gas chromatographic experiments.

\section{Results and Discussion}

Initially, both GC/MS and particle beam LC/MS were run in parallel, but after three runs it was determined that particle beam LC/MS gave so much more information than GC/MS that only particle beam LC/MS was continued. The samples run on the gas chromatograph/mass spectrometer were the polyimide forms of BPDA-PDA, BPDA-PDA-ODA, and BPDA-PDA6FDA. Particle beam IC/MS was also run on BTDAAPB, PMDA-ODA meta-ethyl ester, and unesterified PMDA-ODA polyimide. For BPDA-PDA-6FDA, BPDA-PDA, BTDA-APB, PMDA-ODA meta-ethyl ester, and unesterified PMDA-ODA, both the imide and acid forms were run to see if this technique could distinguish between the two.

The first polymer examined was the acid and imide forms of BPDA-PDA. These two spectra looked virtually identical. Figure 1 shows that after hydrolysis there are two main components. The first is the BPDA tetra acid, which is actually in the ammoniated form in solution but has been drawn as the tetra acid for convenience. The second product is the diamine PDA. When $1 \mu \mathrm{L}$ of this solution was injected directly onto the gas chromatograph/mass spectrometer column no peaks were seen, but when a 1:1 extract with ethyl ether was run, the resulting total ion chromatograph showed only one peak which corresponded to the PDA amine at $m / z$ 108. Figure 2 shows a direct 10 $\mu \mathrm{L}$ injection of the aqueous ammonium solution di-

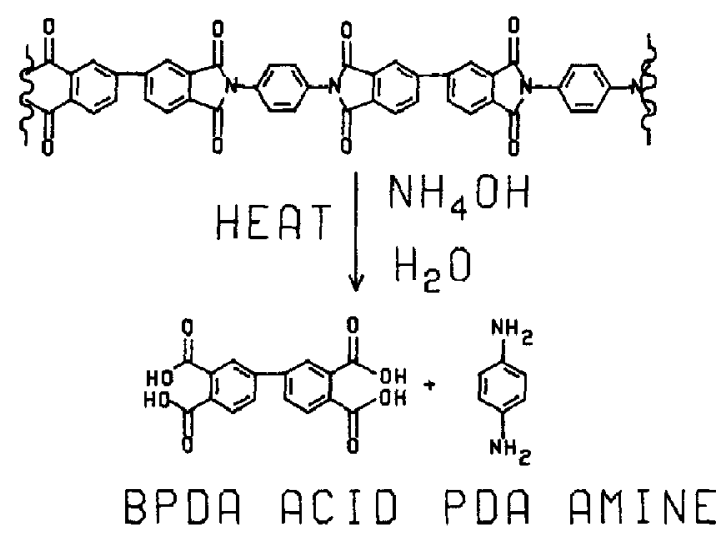

Figure 1. Hydrolysis reaction of BPDA-PDA polyimide with aqueons $\mathrm{NH}_{4} \mathrm{OH}$. 


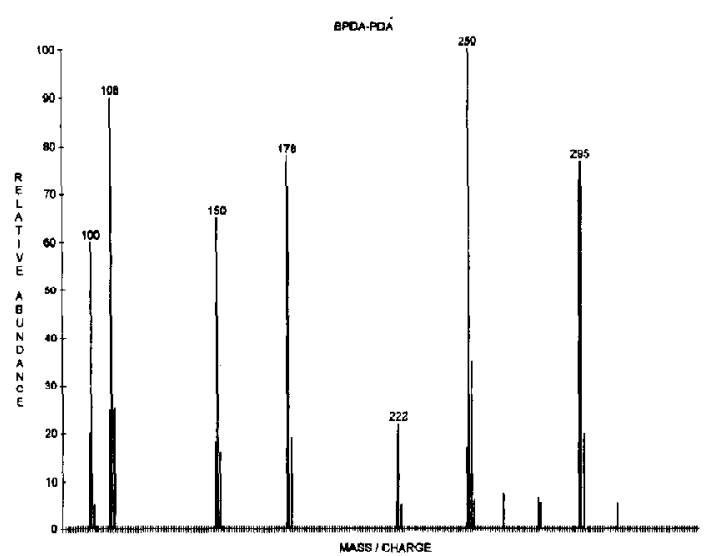

Figure 2. Particle beam spectra of the hydrolysis products from BPDA-PDA polyimide.

rectly into the particle beam liquid chromatograph/ mass spectrometer without further sample preparation.

The particle beam LC mass spectra (Figure 2) show many more peaks than the GC/MS trace. These other peaks range in mass from 100 to 295 amu and can be explained based on a proposed fragmentation pattern for the tetra acid BPDA. BPDA tetra acid has a molecular weight of $330 \mathrm{amu}$ and is conspicuously missing from the mass spectrum. This is not terribly surprising as it should quickly dehydrate to form the dianhydride (294 amu) [10] which is seen as a large peak and protunated dianhydride (295 amu). These types of aromatic and aliphatic anhydrides are known to decarboxylate, expunge $\mathrm{CO}$ and, in the case of aliphatic anhydrides, small hydrocarbons. Because this molecule is aromatic with no aliphatic side chains, it would be expected to lose both $\mathrm{CO}_{2}$ and $\mathrm{CO}$ with no aliphatic moieties being expunged. In fact, $m / z 250$ results from decarboxylation of the parent anhydride ion and $\mathrm{m} / \mathrm{z}$ 222 to an additional loss of a $\mathrm{CO}$ molecule.

As can be seen from Figure 3 , the other peaks in the mass spectrum can also be explained based on $\mathrm{CO}_{2}$ and $\mathrm{CO}$ losses from the anhydride. One might also

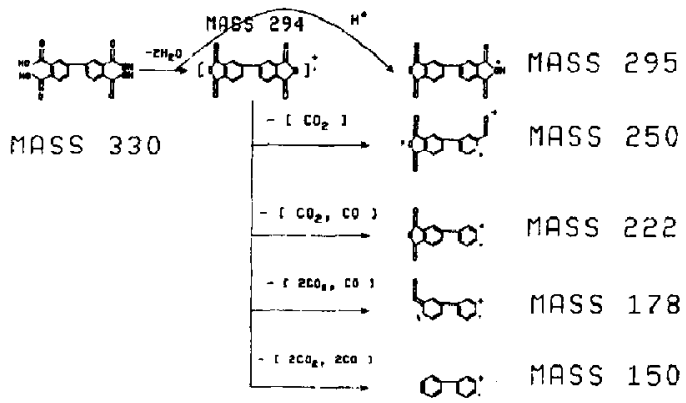

Figure 3. Mass spectral interpretation of the fragmentation from Figure 2, BPDA-PDA hydrolysis spectra.

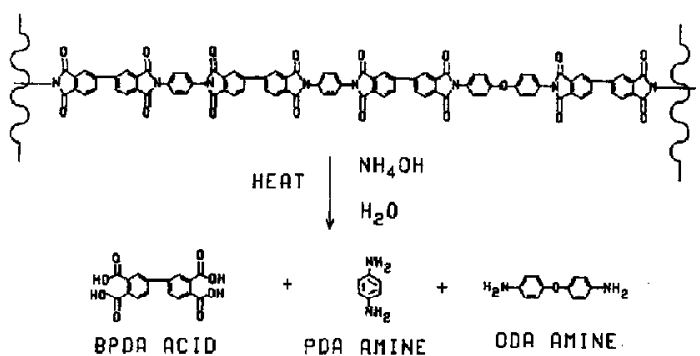

Figure 4. Hydrolysis reaction of BPDA-PDA-ODA polyimide with aqueous $\mathrm{NH}_{4} \mathrm{OH}$.

expect the molecule, given sufficient energy, to fragment via cleavage of the two aromatic rings followed by $\mathrm{CO}_{2}$ and $\mathrm{CO}$ loss, but lack of any peaks at appropriate masses rules out this fragmentation mechanism. The only other major peaks shown in Figure 2 are at $m / z 108$, which corresponds to the PDA molecular ion, and $\mathrm{m} / \mathrm{z} 100$ (protonated N-methyl-2-pyrrolidone), which arises from the solvent contained in the polymer that was hydrolyzed.

Figure 4 shows the hydrolysis reaction for a mixture of BPDA-PDA-ODA polyimide. The GC mass spectra show the two amine hydrolysis products PDA (108 amu) and ODA (200 amu) but, again, do not show the acid moiety. On the other hand, the particle beam spectra show both the amine and acid portions for all the components. The fragmentation observed is, again, consistent with the BPDA-PDA-ODA structure. A comparison of the BPDA-PDA and the BPDA-PDAODA spectra shows that the BPDA-PDA-ODA spectra have only one additional peak, at $200 \mathrm{amu}$, which corresponds to the ODA amine molecular inn. The rest of the spectra can be explained, as in Figure 2, in terms of the BPDA-PDA backbone (Figure 3). Only the imide form of this polymer was available and, thus, the poly(amic acid) spectra were not run; even though the acid form of the polymer was not run, it would be expected to look identical to the imide form as the hydrolysis products should be the same for both polymers.

The last polymer studied by both GC/MS and particle beam LC/MS was a mixture of BPDA-PDA-6FDA. The polymer and its expected hydrolysis products are shown in Figure 5. The GC/MS is capable of detecting both of the amines but, again, not the acid portion of the polymer. A comparison of the relative intensities of the two amine peaks in the GC/MS chromatogram is interesting in that the $6 \mathrm{FDA}$ diamine is only present in a relatively low concentration relative to the PDA in the bulk polymer but, in fact, the 6FDA amine is about ten times higher than the PDA in the GC/MS chromatogram. This is probably due to the differences in solubility of the two amines in ethyl ether coupled with their respective ionization cross sections. Therefore, the GC/MS analysis is not only unable to detect the acid portion of the polymer, but when more than 


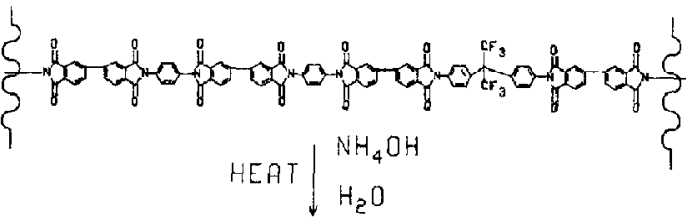

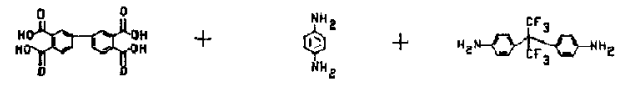

$$
\begin{aligned}
& \text { BPDA aCID PDA AMINE GFDA aMINE }
\end{aligned}
$$

Figure 5. Hydrolysis reaction of BPDA-PDA-6FDA polyimide with aqueous $\mathrm{NH}_{4} \mathrm{OH}$.

one amine is identified one must be careful of drawing conclusions about the relative concentrations of these amines in the polymer from the mass spectral data.

The particle beam spectra for both the imide and acid, as is expected, look similar; the acid and amine portions can both easily be seen, and the intensity of the PDA is much higher than the 6FDA, as would be expected based on their relative concentrations in the bulk polymer. The fragmentation patterns seen in the particle beam spectra are very similar to those of BPDA-PDA (Figures 2 and 3), with the addition of peaks at $m / z 334$ and 265 from the 6FDA ion.

So far, a number of different amines have been examined, but only one acid, the BPDA monomer; the next two polymers contain a different acid monomer, PMDA, which has been reacted with the diamine ODA to form a PMDA-ODA backbone, but the first polymer is the unesterified poly(amic acid) (Figure 6a), while the second poly(amic acid) has an ethyl ester meta to the amide bond (Figure $6 \mathrm{~b}$ ). When this polymer is cured it will form an imide identical to the unesterified acid because it eliminates the ethyl ester as ethanol

UNESTERIFIED POLY(AMIC ACID)<smiles>CNc1cc(C(=O)Nc2ccc(Oc3ccc(NC(=O)c4cc(C(=O)Nc5ccc(Oc6ccc(C)cc6)cc5)c(C(=O)O)cc4C(=O)O)cc3)cc2)c(C(=O)O)cc1C(=O)O</smiles>

ESFERIFIED POLY (AMIC ACID)

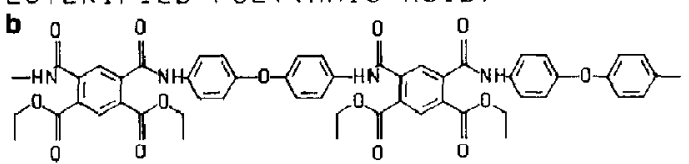

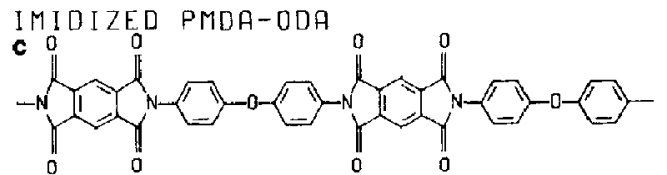

Figure 6. Structures for PMDA-ODA polymers. Unesterified PMDA-ODA (a), esterified PMDA-ODA (b), and imidized esterified and unesterified PMDA-ODA polymer (c). during the curing process (Figure $6 c$ ).

When these polymers are hydrolyzed and run through the particle beam interface into the mass spectrometer, the resulting four spectra look very similar (Figure 7), with only minor variations from spectra to spectra. All the spectra also show a peak at $m / z 149$. This peak is not easily explained in terms of the fragmentation from either the PMDA or ODA and is probably caused by an impurity, phthalic anhydride, in the starting material. The nonimidized poly(amic acid ester) shows no fragmentation indicative of the ethyl esters in the mass spectra. The lack of any detectable differences in the imide and acid form of this polymer is probably due to the evaporation step just after hydrolysis. Although hydrolysis of the ester should generate free ethanol, the evaporation step allows this ethanol to evaporate along with water and $\mathrm{NH}_{4} \mathrm{OH}$.

Figure 8 shows possible structures for the major peaks in Figure 7. Once again, the parent ion of the tetra acid is missing, as expected, but the dianhydride and protonated dianhydride are seen just as with the BPDA acid monomer. The main fragmentation pathway is again decarboxylation and decarbonylation of the aromatic dianhydride. The amine also shows a propensity for fragmentation by cleaving the aromatic ether bond, giving a resulting ion at $m / z$ 108. This fragment was difficult to detect in the BPDA-PDAODA polymer since PDA's major ion is also at mass 108.

In BPDA-PDA-ODA the ODA may be fragmenting to form an ion at $m / z 108$, but the major peak from PDA also occurs at this mass in the form of $\mathrm{NH}_{2}\left(\mathrm{C}_{6} \mathrm{H}_{6}\right) \mathrm{NH}_{2}$. High resolution spectra would have to be acquired to distinguish it from the $\mathrm{O}_{6}\left(\mathrm{C}_{6} \mathrm{H}_{6}\right) \mathrm{O}$ fragment from ODA. The PMDA-ODA polymer does not suffer from the same interference because it does not contain any PDA in the bulk polymer. Therefore, in the PMDA-ODA polymer, the peak seen at $\mathrm{m} / \mathrm{z} 108$ can be attributed to the fragmentation of the ODA molecular ion.

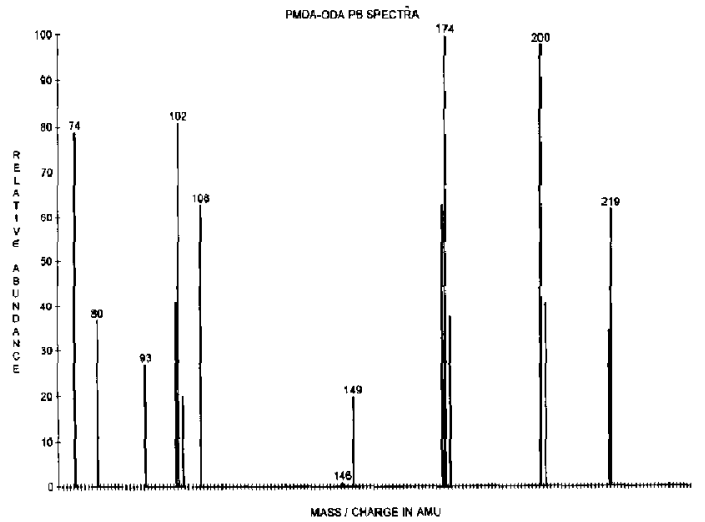

Figure 7. Particle beam spectra of PMDA-ODA. 


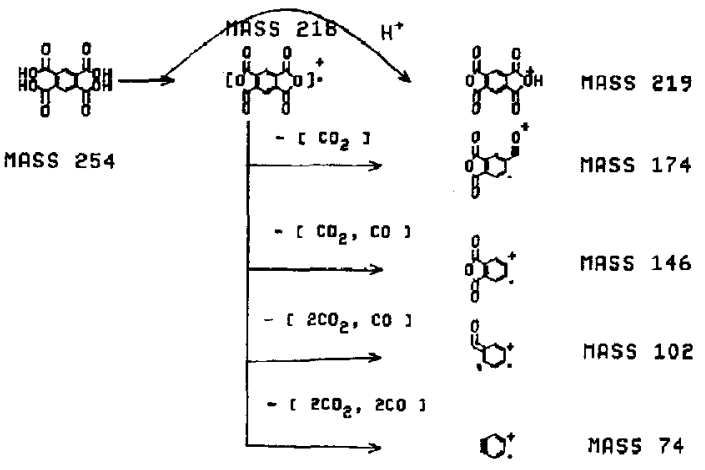

Figure 8. Mass spectral interpretation of the fragmentation from Figure 7, PMDA-ODA polymers.

To try yet another acid amine pair, both the imidized and acid forms of BTDA-APB were hydrolyzed (Figure 9) and the corresponding particle beam LC mass spectra (Figure 10) reveal a number of peaks. These peaks can be explained based on the fragmentation of the BTDA ion which fragments in a manner similar to both the BPDA and PMDA ions. The major pathway involves decarboxylation and decarbonylation until all the carbonyl groups are removed. The BTDA ion also fragments to cleave the benzophenone benzene carbonyl bond, thus breaking apart the two aromatic rings. A close look at the acid and imide spectra reveals no differences in the mass spectra of the hydrolysis products between the two. This is as expected based on their comparable structures.

Of all the polymers studied in this article, PMDAODA is the only polymer that has comparable data in the literature. Therefore, it is interesting to compare the information obtained from the static SIMS work and the present results. The static secondary ionization mass spectra contained peaks at $m / z 219,200,108$, and 100 in an unimidized film but only the $m / z 108$ peak was retained when the film was cured to $400{ }^{\circ} \mathrm{C}$.<smiles>Cc1ccc(Oc2ccc(N3C(=O)C4C(C(=O)c5ccc6c(c5)C(=O)N(C)C6=O)C=CC4(c4ccc(Oc5ccc(C)cc5)cc4)C3=O)cc2)cc1</smiles>

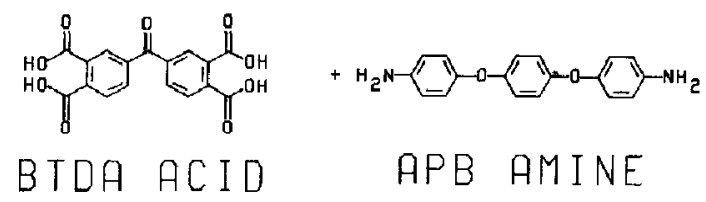

Figure 9. Hydrolysis reaction of BTDA-APB polyimide with aqueous $\mathrm{NH}_{4} \mathrm{OH}$.

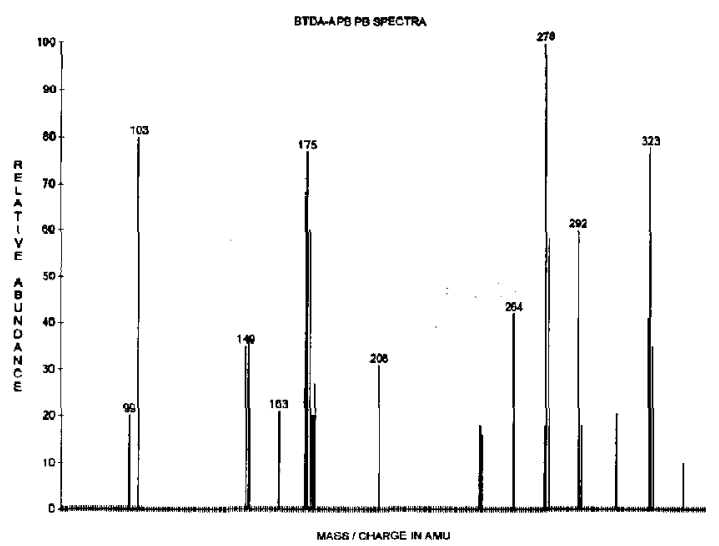

Figure 10. Particle beam spectra of the hydrolysis products from BTDA-APB polymer.

The unimidized spectra are very similar to the spectra contained in this study with the exception that the static SIMS, with its softer ionization method, did not cause the parent acid, at $m / z 219$, to undergo further fragmentation. It is also interesting to note that although the static SIMS data were taken on unhydrolyzed polymer, no high mass PMDA-ODA fragments were seen.

\section{Conclusion}

A comparison is made between the types of information that can be obtained from hydrolyzed polyimides and poly(amic acids) run on a particle beam liquid chromatograph/mass spectrometer versus a gas chromatograph/mass spectrometer. The gas chromatograph/mass spectrometer could not detect either the amine or acid portion of the hydrolysis products when the sample was injected directly into the gas chromatograph. If an ethyl ether extract of the sample is prepared and this ether extract is injected onto the gas chromatographic column, then the amine portion of the polymer could be detected. Detection of the acid portion would require derivatization to more volatile species, but this was not done for these samples.

By contrast, both the amine and acid portions of the hydrolysis products were detected using the particle beam interface without the need for extractions or derivatization. A host of polyamic acids and imides were run to determine the range of acids and amines that could be detected. In all the cases studied, the acid and amine portion of the hydrolyzed polymers were detected.

\section{References}

1. (a) Recent Adrances in Polyimide Science and Technology; Weber, W. D.; Gupta, M. R., Eds. Mid-Hudson, SPE, March 1987. (b) Alston, W. B.; Gratz, R. F. Proceedings of the First 
Pacific Polymer Conference; NASA Technical Memorandum $102353,1989$.

2. Johnson, E. T. J. Appl. Polym. Sci. 1992, 44, 1905.

3. Goldfarb, I. J.; Jones, E. G. Polymer Preprints 1985, 269.

4. Otis, C. E. Appl. Phys. B 1989, 49, 455.

5. Creasy, W. R.; Brenna, J. T. Chem. Phys. 1988, 126, 453.

6. (a) Eldridge, B. N.; Feger, C.; Goldberg, M. J.; Reuter, W.; Scilla, G. J. Macromolecules 1991, 24, 3209. (b) Dugger, D. C.; Stern, M. B.; Rubico, T. M. Mat. Res. Soc. Symp. Proc. 1989, $147,385$.

7. Chace, M.; Plat, M. Proceedings of the 37th Annual ASMS Conference on Mass Spectrometry and Allied Topics; 1989; 264.
8. Linde, H. G.; Meeks, Jr., S. Anal. Chem. 1983, 55, 1443.

9. (a) Willoughby, R. C.; Browner, R. F. Anal. Chem. 1984, 56, 2626. (b) Brown, R. F.; Draper, W. M. In Liquid Chromatography/Mass Spectrometry: Applications in Agricultural, Pharmaceutical, and Environmental Chemistry; Brown, M., Ed. ACS Symposium Series, No. 420; Washington, DC, 1990; p 198. (c) Beir, M. E.; Winkler, P. C.; Herron, J. R. J. Am. Soc. Mass Spectrom. 1993, 4, 38.

10. McLafferty, F. W. Interpretation of Mass Spectra. University Science Books, 1980; p 206. 